\title{
Forum
}

\section{Die erste Preisverleihung am 1. Dezember 2007 von The Missing Link. Der Preis für Psychoanalyse und ...}

\section{Eine Anerkennung des Psychoanalytischen Seminars} Zürich

\section{Christiane Wolf (Zürich)}

Das Psychoanalytische Seminar Zürich (PSZ) steht in der Tradition einer Psychoanalyse, die sich im Spannungsfeld von anderen Gebieten sieht und die Beschäftigung mit Kultur- und Gesellschaftstheorie, mit Kunst und Wissenschaft und mit aktuellen Fragen zu ihrem Selbstverständnis zählt.

In den letzten Jahren hat der gesellschaftliche Druck auf die Psychoanalyse, sich als Behandlungsmethode zu legitimieren, stetig zugenommen. Gesetzliche Regelungen engen die Zulassung zur psychoanalytischen Ausbildung ein auf Ärzte und Psychologen. Die Psychoanalyse wird mehr und mehr zu einer rein klinischen Disziplin.

Wie kann es gelingen, die uns begründende Tradition wieder in den eigenen und öffentlichen Blick zu rücken? Mit einem Preis für interdisziplinäre Arbeiten, welche die Psychoanalyse in Austausch bringen mit anderen Wissenschaften und anderen kulturellen Bereichen! Die Idee war zündend und das 30jährige Bestehen des Psychoanalytischen Seminars Zürich Anlass genug, die Ärmel hochzukrempeln und das Projekt zu verwirklichen.

The Missing Link. Preis für Psychoanalyse und ... wurde 2007 erstmalig vergeben und wird - dank erfolgreicher Sponsorensuche - alle zwei Jahre verliehen werden.

Nach der Ausschreibung gingen 54 hochqualifizierte Bewerbungen ein. Sie deckten ein breites Spektrum verschiedenster Disziplinen ab. Von der 
Quantenphysik über die Mathematik bis zur Philosophie, aus der bildenden Kunst, aber auch vom Theater und vom Film.

Die fünfköpfige Jury - Insa Härtel, Kulturwissenschaftlerin aus Bremen, Bernd Schwibs, Chefredakteur der Psyche, Sönke Gau, Kurator an der Shedhalle Zürich, Vera Saller und Olaf Knellessen vom Psychoanalytischen Seminar Zürich wählte Robert Pfaller zum ersten Preisträger. Preisgekrönt wurde sein Buch Die Illusionen der anderen. Über das Lustprinzip in der Kultur. Robert Pfaller lehrt als Professor an der Kunstuniversität Linz und an der Technischen Universität Wien Kulturwissenschaft und Philosophie.

Es gibt Einbildungen, als deren Träger wir uns sehen, Überzeugungen, die wir für uns reklamieren. Die Illusionen der anderen sind schwer greifbare Einbildungen, die keinen erkennbaren Träger haben. Sie werden anderen zugeschrieben, aber niemand bekennt sich selbst zu ihnen. Sie sind von einem entgegengesetzten «besseren Wissen» begleitet. Es handelt sich um einen Glauben, der die Struktur hat: Ich weiss zwar, aber dennoch ... Wie das Lesen des Horoskops, das gerne begleitet wird von der Bemerkung: «Ich weiss, es ist blöd, aber ich muss unbedingt mein Horoskop lesen.» Irgendjemand scheint daran zu glauben, sonst stünde es ja nicht jeden Tag in der Zeitung. Dieses Moment der Distanzierung ist die Form, in der solche Einbildungen erlebt werden. Aber trotz dieser durch Wissen hergestellten Dezentrierung üben die Illusionen der anderen einen unerklärlichen Zwang aus.

Robert Pfaller erkennt in ihnen das Lustprinzip der Kultur. Es sind Glückstechniken und sie sind überall am Werk: in der Alltagskultur, im Sport, in der Kunst, in allen Spielen, in therapeutischen Techniken, in der Erotik.

Seinen Ideen bereitet Pfaller einen breiten und gleichzeitig vielschichtigen theoretischen Boden. Nachdem er zunächst den Begriff der «Interpassivität» entwirft, der vom delegierten Genuss handelt, bezieht er sich auf die Theorie der Verleugnung von Octave Mannoni. Dort werden zwei Existenzformen von Überzeugungen unterschieden, der Aberglaube und das Bekenntnis.

Das Modell des Aberglaubens ist das Spiel. Mit Johan Huizingas Theorie des Spiels nähert sich Pfaller der Frage nach den libidoökonomischen Bedingungen des Aberglaubens. Dem Spiel gehen wir mit «heiligem Ernst» nach, obgleich dieser Ernst nicht durch Nützlichkeitserwägungen erklärt werden kann. Wir erleben im Spiel grössere Hingabe als im richtigen Leben, ohne aber das Spiel mit diesem zu verwechseln.

Hier kommt die Ambivalenz ins Spiel. Die Faszinationskraft des Spiels erklärt sich aus der Addition der Affektbeträge von Liebe und Hass, die wir dem 
Spiel entgegenbringen. Wir verachten das Spiel, weil es unsinnig ist. Robert Pfallers Verknüpfung ist die: Im Spiel, im Aberglauben, in den Illusionen der anderen können wir uns für einen Moment von der Last des Ich-Ideals befreien.

Das preisgekrönte Buch ist ein spielfreudiges Werk und mit einem Kaleidoskop vergleichbar, durch das sich immer wieder neue Brechungen und Sichten auf unterschiedlichste Fragen ergeben.

Der Preis wurde am 1. Dezember 2007 in der Shedhalle der Roten Fabrik Zürich überreicht, im Rahmen einer Veranstaltung, die eingerahmt wurde von der Uraufführung der Soundskulptur «9 Psychoanalytiker» des Zürcher Künstlers Nik Emch. Die Shedhalle bot dafür den idealen Raum. Als experimenteller Ausstellungsort versteht sie sich als ein Ort «Dazwischen», der neue Formen von Darstellung sucht. Künstlerische, kulturelle und wissenschaftliche Praktiken sollen in einen gesellschaftlich relevanten Dialog gebracht werden.

Daniel Strassberg vom Psychoanalytisches Seminar Zürich beginnt seine geistreiche Laudatio mit einer Warnung: « Ich möchte Sie vor diesem Buch warnen. Heutzutage muss ja beinahe alles, was Genuss verspricht, mit einer Warnung versehen werden. Dass aber Bücher einen warnenden Aufkleber tragen, ist doch eher ungewöhnlich. Ich möchte diesen schönen, die Lust steigernden Brauch nun auch für Bücher einführen, und empfehlen, dieses Buch nur mit einem Sticker versehen auszuliefern, auf dem es heisst: Achtung: Dieses Buch entlarvt Sie schonungslos!»

Strassberg sieht einen grossen Verdienst der Arbeit von Robert Pfaller darin, dass «sie den Blick auf die eigenen Vorurteile richtet, auf jene kleinen unaufgeklärten Bezirke in unserem eigenen Leben, in denen wir unserem magischen Denken frönen, unsere kleinen Zwänge und Perversionen pflegen, unseren Idiosynkrasien nachhängen, ohne sie weiter zu hinterfragen. Sei es, dass wir Fussballspiele ansehen müssen, die uns bei Lichte betrachtet völlig schnuppe sein könnten, sei es, dass wir Horoskope lesen, an die wir nicht glauben, sei es, dass wir Haaranalysen machen lassen, die wir ohne weiteres als baren Humbug erkennen, sei es, dass wir Diäten befolgen, die jeder wissenschaftlicher Grundlage entbehren, uns aber von Brigitte oder Annabelle empfohlen werden. Alleine schon die Aufmerksamkeit auf diese allgegenwärtigen, aber für gewöhnlich unterbelichteten Phänomene gerichtet zu haben, macht die Lektüre dieses Buches vergnüglich - was für ein wissenschaftliches Buch schon ein Ereignis ist.»

Im Zentrum der Laudatio steht der vorurteilkritischen Ansatz des Buches: «Im Spiel kann ich aus meiner Haut zu schlüpfen, um ein Anderer zu sein, ohne die Retorsion des Ich-Ideals fürchten zu müssen. Und gerade darin liegt die auf- 
klärerische und emanzipatorische Potenz des Vorurteils: dass ich mich vergessen kann, dass ich für einen Moment nicht mein Ich sein muss.» Und weiter: «So werden Vorurteil und Aberglaube, die seit der Aufklärung als die Vehikel der Reaktion und der Lebensfeindlichkeit gelten, in Pfallers alchemistischen Küche zu Trägern der Lust und des Vergnügens in unserer Kultur, allerdings nur solange sie nicht als meine Lust und als mein Vergnügen identifiziert werden können.»

Der Dankesrede von Robert Pfaller (nachfolgend abgedruckt) folgte die Uraufführung der Soundskulptur «9 Psychoanalytiker». Sie brachte die Psychoanalyse und die Kunst in Austausch und liess den zuschauenden Zuhörer an Metamorphosen teilhaben:

Die Uraufführung ist Teil einer Ereigniskette, an deren Anfang Gespräche standen, die der Künstler mit neun Psychoanalytikern und Psychoanalytikerinnen des Psychoanalytischen Seminars Zürich führte. Den Abschluss des Projektes wird eine Ausstellung bilden, die Textmaterial, Bilder und Klangspuren zu einer umfassenden Installation vereint. Den Gesprächen folgte die malerische Umsetzung. Es entstanden neun Bilder, in denen der Künstler seine in den Sitzungen erlebten inneren Vorgänge verbildlichte. Es sind hautfarbene Ölbilder von Körpern, Organen ähnelnden Körperteilen, ausmodellierten Flächen.

Diese Bilder - Porträts und Selbstporträts in einem - stehen zunächst verhüllt vor dem Publikum. Die Soundskulptur entsteht: Die neun Psychoanalytiker treten vor «ihr» Porträt und zeigen sich in ihrer äusseren Erscheinung. Dann enthüllt einer nach dem anderen «sein» Bild und tritt an ein Mikrophon, um Klatschimpulse zu geben. Diese Impulse werden vom Künstler in einen Loop gespeist, sodass sie am immer selben Ort des Kreises hörbar sind. Die neun sich überlagernden Klatschsequenzen sind die Basis für die sich anschliessende musikalische Improvisation, in der Nik Emch die Klänge mittels verschiedener Klangfilter bearbeitet, einem Bildhauer - und, könnte man sagen einem Analytiker - ähnlich, der verdeckendes Material immer feiner abträgt. Auf der akustischen Ebene wiederholt sich der Prozess des Malens, es entstehen Klangbilder, Klangkörper, deren Resonanz man sich als Zuhörer nicht entziehen kann.

Es war der Abschluss der Preisverleihung, die grossen Anklang fand und dem ins Leben gerufenen Preis keinen besseren Start hätte bereiten können. Das Psychoanalytische Seminar Zürich feierte am Abend sein Jubiläum und die erste Preisvergabe mit einem rauschenden Fest.

Pfaller, R. (2002): Die Illusionen der anderen. Über das Lustprinzip in der Kultur. Frankfurt/Main: Edition Suhrkamp. 


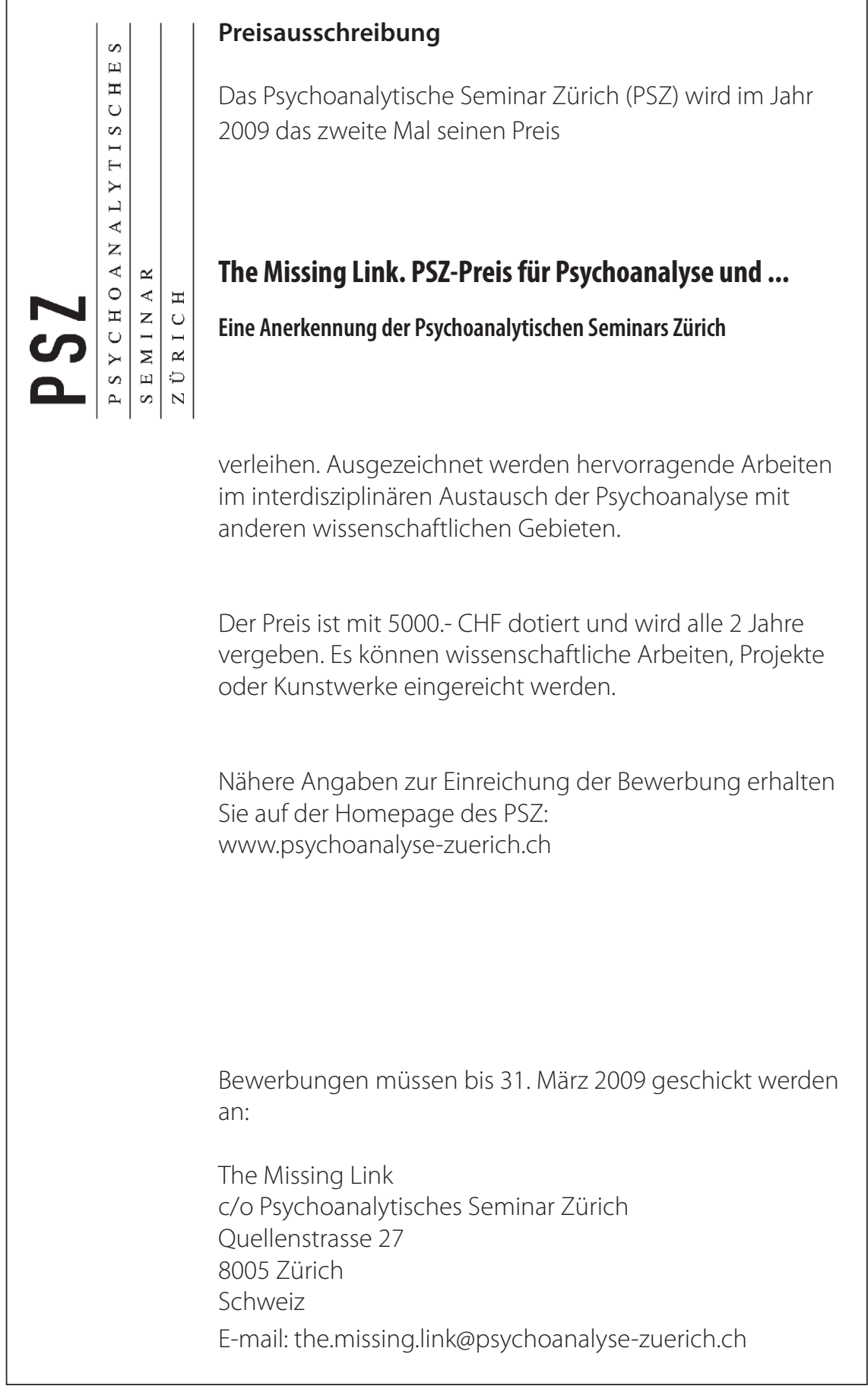

